\title{
Exploring Smoking Ban Adherence among Patients and Prevention and Control Programs at Amanuel Mental Specialized Hospital, Addis Ababa Ethiopia
}

\author{
Abebaw $\mathrm{D}^{1 *}$, Shumet $\mathrm{S}^{1,2}$ and Getnet $\mathrm{M}^{1}$ \\ ${ }^{1}$ Department of Epidemiology, J imma University, \\ Ethiopia \\ ${ }^{2}$ Department of Psychiatry, University of Gondar, \\ Ethiopia \\ *Correspondling author: Dessie Abebaw, Department \\ of Epidemiology, J imma University, Ethiopia
}

Received: August 29, 2017; Accepted: September 25, 2017; Published: October 02, 2017

\begin{abstract}
Background: The implementation of smoke-free policies in mental health settings represents a significant challenge. Timely smoke free environment initiation is the main factor for successful smoking ban program. However studies to understand patients, health care givers and staffs perspective on smoking ban initiation has been limited in Ethiopia. The main aim of this study was exploring smoking ban adherences among patients and prevention and control programs at mental health care institutes.
\end{abstract}

Methods: The in-depth interview was considered to be appropriate for investigating patients, care givers and hospital staffs attitude, practice and hospital smoking ban program related factors. A total of 22 patients who were smokers, 12 patient families and 14 staffs were participated in the study. A thematic content analysis of the interviews was performed using the Open Code software version 3.6.

Result: We found that having different sources of cigarette access, less group therapy or psychological treatment and cigarette contraband were the main reasons for less initiation of smoking ban adherence. On the other hand, Staffs were more motivated for initiation of smoking ban program.

Conclusion: Challenges of contrabands, low psychological treatment, having different source of cigarette and poor occupational therapy were factors that most influenced timely smoke free environment initiation.

Keywords: Adherence; Mental specialized hospital; Total smoking ban

\section{Introduction}

Patients, who smoke at heavier rates than in the general population and most patients, start smoking in their teens, before the illness begins. This excessive smoking is frequently attributed to the high mortality and morbidity rates among this particular group of people. Around the world, at the present time, it is estimated that tobacco kills over 4.9 million people per year around the world [1-5]

Smoking is one of the known causes of chronic health conditions. Chronic health conditions, like cancer, diabetes, or heart disease, in turn are primary drivers of health care spending, disability, and death [6]. Though many countries decide smoking ban in their working and public environment, there was a great debate between public health professionals and policy makers in one group and tobacco industries and organizations supported by tobacco industries in another group [7].

In psychiatry hospital Tobacco smoking remains a neglected issue despite high rates of associated morbidity and mortality. Mental health facilities are highly neglected to control smoking as compared to general population and other facilities $[8,9]$. Smoking also appears to play a central role on social interaction on patient wards. Like many policies, smoke-free policy implementation is a process, not an event. Evidence of problems does not mean the policy is inappropriate or a failure. Addressing the damage caused by tobacco for people with mental disorder requires a multipronged approach across the continuum of care $[1,10]$.

Programs which support smoke cessation at psychiatry health facilities during patient stay have both an efficacious and cost effective $[11,12]$. In terms of lives saved, quality of life, and cost efficacy, treating smoking is considered to be the most important activity a clinician can undertake [13-15].

It takes courage, leadership and planning to successfully implement a smoke-free policy in mental health settings. Management and clinicians should work closely together to develop and coordinate the implementation strategy, ensuring that resources are effectively used and deadlines are met. Key success factors are effective management at both central and local levels, as well as consultation with service users, careers and staff to gain support for the policy and obtain suggestions for improvement. Other important factors are advance planning, recruitment of experienced staff, effective communication and extensive training of staff in smoking cessation support $[2,12]$.

Although in psychiatry hospitals total smoking ban was not strictly enforced and was overall acceptable to both patients and staff, total ban increased the proportion of smokers who tried to quit smoking during their hospital stay $[8,11,16,17]$. This is also supported in other
J Fam Med - Volume 4 Issue 6 - 2017

ISSN : 2380-0658 | www.austinpublishinggroup.com Abebaw et al. (@) All rights are reserved
Citation: Abebaw D, Shumet S and Getnet M. Exploring Smoking Ban Adherence among Patients and Prevention and Control Programs at Amanuel Mental Specialized Hospital, Addis Ababa Ethiopia. J Fam Med. 2017; 4(6): 1129. 
facilities like in universities above $45 \%$ of the students are agreed for total smoking ban in the university [16]. Psychiatric institutions that implement general smoking policies must be aware that they need to overcome the problems of compliance and inadequate smoking rooms, otherwise the problem of environmental tobacco smoking exposure is not adequately solved $[1,12,16]$.

Mental healthcare staffs are more than twice as likely to be smokers as their colleagues in general healthcare. Mental healthcare staffs are also known to be significantly less positive towards smokingrelated policies and treatments than their counterparts in other areas of healthcare. A recent UK survey reported that roughly 1 in 10 staff in general healthcare settings disagreed with a smoking-ban in their wards or clinics; however, 1 in 3 psychiatric staff were against such a ban in their settings $[18,19]$.

In Ireland, Scotland and UK smoke-free legislation has been introduced which bans smoking in enclosed public areas or workplaces. In Scotland and Ireland, mental health units are exempt from implementing smoke-free legislation. Guidance for voluntary smoke-free implementation in mental health units is available for Scotland and for Ireland $[19,20]$.

These fears are understandable but research evidence does not support them. A review of 22 studies of total and partial smoking bans in mental health settings found no adverse effects in terms of unrest or refusal to comply reviewed 26 international studies of smokingbans in mental health units, finding no increase in aggression, use of seclusion, discharge against medical advice or use of medication on an 'as required' basis [12].

Staff attitudes can become more positive, who found that initially only $7 \%$ of staff favored a smoking ban before its introduction, rising to $90 \%$ post-implementation. In a Canadian study, successful smokefree implementation was found to depend on staff preventing patients from gaining access to tobacco $[8,15]$.

In Ethiopia mental health hospital, majority of the patients were smokers, the newly admitted mentally ill patients are exposed to smoke cigarette thought, and previously they were not smokers. The Ethiopian government declare smoking ban in public and working areas by 2014 and Amanuel mental specialized hospital were the first working environment to implement this regulation. The hospital develops its regulations to have successful smoking ban program. Inconsistent adherence to the smoking ban and prevention program might lead to ignoring smoking ban activities and, making it difficult to achieve high success rate. Therefore this study helps to understand what factors influence the willingness or the ability of patients to adhere to smoking ban and prevention programs, to utilize this understanding to change the affected individual's behavior to improve adherence rates and, to modify the existing programs. On the top of this, the study would motivate the health authorities and policy makers to have close contact with health institutions to decrease cigarette smoking activities and mobilizing affected individuals in order to adhere the smoking ban program.

\section{Methods}

\section{Study setting}

Institution based study was conducted in Amanuel Mental
Specialized Hospital in Addis Ababa. It is one of the oldest hospitals established in 1930 E.C during the Ethio-Italian war and it is the only mental Hospital in Ethiopia. In the Hospital the health service had been given up in 1940 by low level psychiatric professionals. Starting from 1946-1970 the treatment was given by doctors came from Russia, Bulgaria, and Cuba.

It is located in western part of Addis Ababa in Addis Ketema Subcity, kebele 08 . The hospital is working on increasing the efficiency \&effectiveness of mental health care excellences by giving core mental clinical services, conducting research and trainings and other administrative services. More than $41 \%$ of the hospital patients were cigarette smokers.

\section{Study design}

Methodologically, this qualitative study was framed within phenomenology in its natural setting to explore smoker mental patients adherence to total smoking ban and prevention programs in Amanuel mental specialized hospital. This phenomenology study describes the meaning for several individuals of their lived experiences of a concept. The researcher focus on describing what all participants have in common as they experience a phenomenon.

\section{Study participants and procedures}

Adult male and female mentally ill patients admitted in the hospital who were smokers, hospital workers including health professionals' guards and senior managements and patient families were the study participants. Patients who were smokers before three months in which the hospital declared total smoking ban and have an insight to respond were eligible to participate whereas, patient family who were stayed at least three weeks in the hospital were eligible to participant in the study. Saturation of data occurred with a sample of 22 patients who were smokers, 12 patient families and 14 staffs. Participants were selected purposively. Before interview the purpose and importance of this study was explained for each participant, and if they agreed to participate, they signed an informed consent form prepared in Amharic which is the local and national language of Ethiopia, and participants' involvement was assured on voluntary bases. Participants who were unwilling to participate and want to abstain at any step of in-depth interview were informed to do so without any restriction.

\section{The in-depth interview}

In-depth interviews were conducted by one interviewer who is trained in qualitative interviewing techniques using Semi-structured guide with one note takers. The guide was prepared in English and translates in to Amharic which is the local language. Interviews were undertaken by audiotape, by note taking and again by observation of the participants during the interviewing. The interviewer was, guided by the participant's responses in deciding when and how to probe the emergent themes. The interviews lasted averagely 50-60 minutes and the audio tape record was done with the consent of the interviewees. The interviews were continued until all categories are addressed and point of saturation reached.

\section{Data analysis}

Tape recorded in-depth interview were firstly transcribed in Amharic character and then translated in to English by two trained staffs in Amanuel mental specialized hospital. Codes were developed 
based on original terms used by participants. The translated data was read repeatedly in order to have clear understanding of the idea and to categories for appropriate themes. The transcript and notes were analyzed using open code version 3.6 then the data were cleaned, saved in plain text file. A descriptive phase of identifying meaning units and assigning codes which were then compared and reorganized into tentative categories.

\section{Ethical consideration}

Ethical approval was obtained from ethical review committee in Amanuel Mental Specialized hospital. To insure the ethical issues not to be violated, written consent was taken from each interviewee. In similar ways, the recordings and notes has been kept in a safe and protected place to assure privacy.

\section{Findings}

\section{Characteristics of the study participants}

A total of 22 ( 6 females and 16 males) patients who were smokers, 12 patient families ( 4 females and 8 males) and 14 staffs ( 5 females and 9 males) a total of 48 participants were participated in the study. Majority of patient families were stayed above 25 days in the hospital. Three main themes which emerged from the content analysis are reported in detail below.

\section{Theme one: patient related factors}

Patients' attitude towards smoking ban: Temporarily the Ethiopian government declared smoking in public areas, health institution and other working areas is totally forbidden. Following this, Amanuel mental specialized hospital applies the rule immediately. All participants accept total smoking ban program but, practically less than half of the participants were never smoke cigarette and advice others not to smoke after the hospital declared total smoking ban.

One patient who stayed for 35 days in the hospital explains his feeling about the hospital smoking ban program and his commitment as follow.

".....Really it is wonderful; I can say it was late. From the beginning any patient admitted to hospital should obey the rules and regulations of the hospital and the order of the doctor. So after smoking ban I never smoke and I strongly fight those who smoke cigarette in my case team".

A 45 years old patient who was smoker for 15 years explains his opinion that total smoking ban is not difficult as everybody thinks.

"I was chronically dependent on tobacco, but its effect on my health becomes worse and worse. That is why I am admitted here. I decide to stop smoking as soon as I hear the hospital has declared total smoking ban. Then I tried to stop smoking and still I don't smoke as well I don't face any problem starting from the beginning of smoking ban so that it was not difficult as they think; it is possible to stop smoking."

On the other hand ,majority of the participants are smoking at least once a week because of different reasons, like peer pressure, availability of cigarettes in the hospital that inters via patient family, visitors, patient themselves and the absence of organized rehabilitation center (Table 1).

Expecting psychological health problem: Majority of the respondents do not face any psychological health problem. But some
Table 1: Distribution of patients' acceptance towards smoking ban program and their adherence in Amanuel mental specialized hospital 2014 G.C $(n=22)$.

\begin{tabular}{|c|r|c|}
\hline Main themes & Sub themes & Number of participants \\
\hline \multirow{3}{*}{ Patients attitude } & Support total smoking ban program & 22 \\
\cline { 2 - 3 } & Support smoking ban at once & 20 \\
\cline { 2 - 3 } & Support smoking ban step by step & 2 \\
\hline \multirow{2}{*}{ Adherence } & Still smoking & 13 \\
\cline { 2 - 3 } & Totally stop smoking & 9 \\
\hline
\end{tabular}

Table 2: Source of cigarette where patients get cigarette, Amanuel mental specialized hospital, $2014(n=22)$

\begin{tabular}{|c|c|c|}
\hline Main theme & Sub themes & Number of patients respond \\
\hline \multirow{3}{*}{ Source of cigarette } & From new comer patient & 6 \\
\cline { 2 - 3 } & From patient family & 6 \\
\cline { 2 - 3 } & From patient him self & 4 \\
\cline { 2 - 3 } & From peers & 6 \\
\cline { 2 - 3 } & From out patient & 2 \\
\hline
\end{tabular}

of them said they fill badly when they see somebody was smoking. And, they developed some disturbance in their life. One participant who stops smoking describes the psychological health effect of smoking ban for question: is there any health problem you face because of you do not smoke after the hospital has declared total smoking ban in the hospital ground?

".......yes, frankly speaking they are few patients to decide total smoking ban. At the evening the hospital securities/guards do not controlled while patients are smoking. As a result, they bring cigarette easily outside the hospital and they smoke at night which disturbs my life because the smell of cigarette affects my health."

\section{Theme two: source of cigarette affecting adherence}

Majority of the patient respondents agreed that availability of cigarettes in the hospital influences smoking ban adherences. They explained that mainly cigarettes are available by new comer patients, patient family, peers and by patient him/her self. Patient families bring cigarettes to the hospital by their socks, capes and inside the bread and they were sold to patient (Table 2).

A patient whose age was 45 years old and stayed in the hospital for 29 days explains how cigarettes are available in the hospital and it affects smoking ban adherences:

"... Mostly patient families bring cigarette outside the hospital and they sold for patients. They know it is risky if somebody gets them. There for they always give to one patient to sell for other patients. On the top of these, patients in the substance ward begin to sell to other patients at the evening because at the evening there is weak control from the security guards. Therefore patients change their cloth to bring cigarette then can have cigarette access easily."

Another a 30 years old female patient who was stayed for two months in the hospital explains the reason why she was unable to stop smoking and where she gets;

"Before this smoking ban program, I was addicted to smoking. After the hospital declares the regulation, I stop smoking cigarette for 14 day. On the top of this, security guards work strongly on preventing 
Table 3: Staffs attitude and practice towards total smoking ban at Amanuel Mental Specialized hospital, 2014 ( $n=14)$.

\begin{tabular}{|l|l|l|}
\hline \multicolumn{1}{|c|}{ Main themes } & \multicolumn{1}{c|}{ Sub themes } \\
\hline \multirow{3}{*}{ Staffs attitude towards smoking ban } & Support total smoking ban & \multicolumn{1}{|c|}{ Number of participants } \\
\cline { 2 - 3 } & Do not support total smoking ban \\
\cline { 2 - 3 } & understand total smoking ban creates safe working environment \\
\hline \multirow{3}{*}{ Staffs practice on controlling smoking } & Advice and control the client while they are smoking \\
\hline & counseling and educating by program in the ward \\
\hline & Reporting to smoking ban committee \\
\hline
\end{tabular}

entrance of cigarettes. But they didn't continue their commitment especially at evening. With this availability of cigarette in the hospital is increased. If there are one or two cigarettes from one smoker everybody fight with these two cigarettes. These cigarettes are available due to patient families and new patients bring the cigarette and more than 10 patients fight with these cigarettes. With this, I fail to stop smoking"

\section{Theme three: staff and patient family attitude and practice}

Patient family attitude and practice: Majority of patient family respondents agreed that new comer patient families were the main source cigarettes. On the other hand, majority the respondents decided that as the patient family is waiting in the hospital they do have positive attitude towards total smoking ban in the hospital. A 40 years old women whose son was smoker before smoking ban explained her attitude and commitments as follow:

"... Early cigarette was sold freely. But now it is decreasing. Before this time we families are ordered by our patient to bring cigarette. At this time total smoking ban helps us not to be ordered. Therefore, it is appreciable rule to keep the safety of our patient". In addition to this, I always give advice the patients while they are smoking and I inform for nurses and security guards while families bring cigarettes and patients are smoking in latrines and corridors.

The other patient family also responds how they protect patients from smoking and how they fight the entrance of cigarette to the hospital:

"... There is one patient who jumps through the window to bring cigarette outside the hospital and to sell for other patients. But, I always cheek his pocket and I take the cigarette from him to give cigarettes to concerned body like nurses, smoking ban committee and hospital security guards. By now this patient becomes comfortable and by this time he doesn't smoke. I try my best as much as possible what I can. I would like to say thank you what a hospital takes an action for total smoking ban".

\section{Staff attitudes and practice}

There are two main sub themes which influences total smoking ban. These are (1) Staffs attitude towards total smoking ban (2) staffs practice towards total smoking ban

Staffs attitude: Table 3 staffs attitude and practice towards total smoking ban at Amanuel Mental Specialized hospital, $2014(\mathrm{n}=14)$.

Majority of the staffs support total smoking ban and all of the staff respondents understand total smoking ban creates safe working environment. One psychiatrist who was working in the hospital for 20 years explained his opinion as follow:
"...This action is the most interesting. When I hear the hospital declare total smoking ban I become happy, because the hospital compound becomes worse and worse due to smoking and all staffs are passive smokers. Previously patients smoke freely in the ward and those patients who do not smoke always disturbed by it. In addition to this, some patient families' reports for us our son tries to smoke cigarette because of all patients smoke freely in the ward. But now it is not true. Personally I support total smoking ban".

On the other hand some respondents do not support total smoking ban before any psychotherapy treatment. A female respondent who do not support total smoking ban in the hospital said that:

"I appreciate smoking ban in the hospital. But I don't support total smoking ban action because they consider smoking cigarette as self medication. Before launching total smoking ban, the hospital should give psychotherapy for the smokers and then they will prepare theme selves to stop smoking."

Again a male psychiatrist respondent who was working in the hospital for 10 years explained his opinion why he was not support total smoking ban:

In our hospital the management have decide smoking ban in the hospital. I have two opinions concerning the smoking ban. Stopping cigarette smoke is appreciable but, it is performed not based on it's in sequential. First education or psychotherapy treatment must be given, but it is not done. Here patients should give time to stop smoking. But some persons order patients if you smoke cigarette you will discharge and others warning them, this motivates to smoke some patients who do not like to wait in the hospital".

The activities of staffs is the most important to be successful for total smoking ban. For this program almost all staffs perform their responsibility. How staffs perform their activities is narrated as follow.

A respondent who support total smoking ban explain his commitment or activities as follow:

"Since I am health professional I educate the smokers how cigarette affects life. On the top of this, some patients smoke in the corridor, latrine and showers room; when we say it is forbidden to smoke in this place they think it is possible to smoke on other places. Therefore we educate simply how cigarette smoking affects our life; even you will take a drug with high dose if you smoke cigarette. And also some families bring cigarette due to their patient forced to bring outside the hospital. So we educate the families."

Controlling the entrance of cigarette is the most important point to be successful total smoking ban. One respondent narrates how they 
control the entrance of cigarette to the hospital as follow.

"The first action should avoid the entrance of cigarette to the hospital. Cigarette enters by patient families, extra person and sometimes patients bring cigarette outside the hospital. This is due to they change their cloth and they can go out easily outside the hospital. This is the weakness of the ward nurses to allow their cloth other than their "bijama". So that, every staff should work hand in hand otherwise it will be clumping in one hand. On the top of this, we protect individuals when they are selling cigarette and we inform any information for cigarette smoking ban committee. Here I would like to inform you some staffs inform for the patient he/she has told us you are smoking or selling cigarette. This leads the patient hating the staffs which influence the patient to accept his or her counseling.

\section{Theme four: program related factor}

Participants describe some entertainments like volley ball, chase, tennis ball, movies, dama, television in each ward, programmed films, libraries and others should be available in the hospital. On the top of this, the hospital shall include programmed work activities like compound cleaning program, health education and occupational therapy .almost all the participants agreed that occupational therapy and televisions in each ward were not available .A 50 years old psychiatry nurse who was working for 31 years in the hospital explains what the hospital program incorporate before declaring smoking ban as follow:

"Primarily psychology professionals expected more to give counseling because they can easily understand its benefit and the possibility to avoid smoking. On the top of these, television and other games should available in every ward and any managers should follow its continuity. If we continue with this we will the pillar or reference for other organizations. Additionally, in our hospital there is occupational therapy but, it is still not well organized. The hospital should give more emphasis for this department. Patients should train some occupational activities based on their interest. Then they can easily forget smoking cigarette."

A 26 years old health officer working 4 years in the hospital said the hospital shall include the following programs in order to forget sensitizing cigarette smoking habit:

"Though it is a wonderful program to develop safe working environment for workers patients and patient families, the program miss the required things that supports the patient not to sensitize smoking. So that, to forget one thing there should something that can replace it. In order to strengthen patients to far from smoking there shall be movies, different books library, daily counseling, and strict control on contrabands even discharging after repeated advice when the patient sells cigarettes. If this type of action continue all patients will be strict to stop smoking and the hospital will be safe working environment. Here we should understand drug is not the only treatment".

\section{Discussion}

These results show that a complex set of dilemmas exist in psychiatric settings with regard to smoking. These include balancing the right to smoke, the right to adequate standards of care and the right to safe work practices and environments. The issue of neglect and negligence, duty of care will also be discussed in relation to these results.

After the hospital launched total smoking ban all the respondents support the hospital action .but, less than half of the smokers were never smoke cigarette after total smoking ban. Here the researcher understands that still the majority of the respondents are smoking though they decrease the amount they take per day. This is because before making the transition to a smoke-free environment, consultation or psychotherapy should take place widely with service users.

A qualitative study conducted in England (2006) psychiatry setting on psychiatry patients majority of the smokers do not smoke after the smoke free environment policy is launched for the first four months, but in our hospital majority of them smoke after the hospital launched total smoking ban for the first two months. This is may be due to our hospital do not perform an action before launching total smoking ban. These are may be preparing nicotine replacement therapy/NRT/, providing materials for recreation training or preparing staffs to counsel smokers.

Majority of respondents in all groups said that cigarette enters by new comer patients, patient families, visitors and from inpatient smokers. This influences not only those who are unable to stop smoking but also those who are still do not smoke after smoking free environment policy is launched. Here even the patients decide to stop smoking cigarette they will face problem when they see their peers are smoking together in the latrine, corridors and some other places. This is because availability of cigarette and seeing their peers are smoking relapses again their previous exposure. Therefore to be successful for free smoking environment program, controlling the entrance of cigarette is the pillar action. From this we can understand that the hospital regulation is not performed to control entrance of cigarette or contraband beginning from the gate of hospital, triage and hospital staff activities.

According to the respondents (smokers, patient families, and staffs) availability of entertainment (like volley ball, TV, rehabilitation center, dama, chase, library, group therapy and any other activities) supports them to stop smoking in short period of time. This is because smokers become busy by doing different activities. Specially allowing smokers to spend more time in rehabilitation center helps individuals to create many technical activities that entertain themselves. But according to our observation and respondent view there is no enough materials, trained occupational therapists that can train patients based on their interest. This may be due to either less attention of senior management staffs or low rehabilitation staff motivation.

Concerning the health impact of smokers due to total smoking ban majority of the clients respond that we do not face any health problem rather we fill goodness. A study conducted in Ireland on mental health setting about free smoking environment action and fears of staffs support our result [16].

Patient families are the most important team to stop smoking because they do have close relationship. Almost all respondent families support smoking ban and they try their best to protect smokers from getting cigarette and smoking. This may be the result of education given for them by the hospital staffs and their negative attitude towards cigarette smoking. But the new comer patient 
families come with cigarette and matches to the hospital. This may be because of they do not know launching of total smoking ban and absence or not enough immediate education for them about smoking ban.

In our study majority of respondent staffs have support total smoking ban. But a recent UK survey reported that roughly 1 in 10 staff in general healthcare settings disagreed with a smoking-ban in their wards or clinics; however, 1 in 3 psychiatric staff was against such a ban in their settings [18]. This may be due to staffs in Amanuel hospital less likely smokers than psychiatric staffs in UK.

In this study cigarette enters in to the hospital in different ways for patient smokers. Especially at the evening substance ward patients bring cigarette to the hospital. Then they sell this cigarette to others by five to ten Ethiopian birr. This situation is the major setback for success of free smoking -environment .because when individuals see this situation it relapses again, even they were decide to stop smoking they try to seek any alternative to get cigarette.

This problem may happen with the following reasons; firstly staffs negligence of their responsibility to control smoking. At the evening patients change their cloth then they leave the hospital to bring cigarette. Here if the patients do not get the opportunity to get their normal cloth at the evening other than bejama (hospital uniform) they cannot get out off the hospital. On the top of this, it may be their less sensitive to inform to the security guard as soon as they see. Additionally, not only smoker but also anybody should be seen at the gate of the hospital for the sake of controlling entrance of cigarette. Thirdly it may be psychological therapy do not give for those substance addicted individuals in special case. In a Canadian study, successful smoke-free implementation was found to depend on staff preventing patients from gaining access to tobacco.

Adherence to the total smoking ban program appeared to be better after three weeks of smoking ban declaration. It is because of initially or the first three weeks there was availability of cigarette in the hospital ground and also patient families were not aware the declaration of smoking ban by the hospital.

Smokers who were adhere smoking ban starting from declaration of smoking ban in the hospital ground do not continue their adherence because of the non adhered patients bring cigarette to the hospital and they sell it for the adhered patients. The researcher conclude that challenges of contrabands, low psychological treatment, having different source of cigarette and poor occupational therapy were factors that most influenced timely smoke free environment initiation.

\section{Authors' Information}

The authors (Dessie Abebaw) are public health specialist in biostatistics and epidemiology who is working at Jimma University, department of epidemiology. Shegaye Shumet is psychiatry professional working at university of Gondar, department of psychiatry and Masrie $\mathrm{G}$ is a biostatician who is working at Jimma University, department of epidemiology.

\section{Acknowledgements}

The researchers acknowledge Amanuel Mental Specialized
Hospital for funding the study. I also acknowledge Tariku Jote, Begashaw Ashebir and Degifie Nebie for their coordination during data collection. Finally they appreciate the study participants for their cooperation in providing the necessary information.

\section{Funding}

This research work was funded by Amanuel Mental Specialized Hospital.

\section{References}

1. Hashimoto K, Makinodan M, Matsuda Y, Morimoto T, Ueda S. Smoking bans in mental health hospitals in Japan : barriers to implementation. Ann Gen Psychiatry. 2015; 1-6.

2. Smoking ban policy in Ethiopia by Drug Administration and Control Authority of Ethiopia. 2005.

3. Series GS. Literature Review on the Health Effects of Smoke-free Policies in Light of the WHO FCTC.

4. Johnsen JV, Løken KV, Johnsen JV, Løken KV. Smoking Bans, Materna Smoking and Birth Outcomes. 2012; 7006.

5. Lawn S, Pols R. Smoking bans in psychiatric inpatient settings ? A review of the research. 2005; 39: 866-885.

6. Sturm R. The Effects Of Obesity, Smoking, And Drinking On Medical Problems And Costs. 2002; 21: 245-253.

7. Mckee $\mathrm{M}$, Hogan $\mathrm{H}$, Gilmore A. Why we need to ban smoking in public places now. 2004; 26: 325-326.

8. Etter M, Nawaz A, Etter J. Acceptability and impact of a partial smoking ban followed by a total smoking ban in a psychiatric hospital. 2008; 1-7.

9. Oli D, Lubman DI, Fraser R. settings : a biopsychosocial perspective. 2007.

10. Variations D. GUIDEBOOK: Common Psychosocial Problems of Schoo Aged Youth: Table of Contents Accommodations to Reduce Problems. 1563(310).

11. Tubbs JS, Garner LIZ. Survey of staff attitudes to smoking in a large psychiatric hospital. 2004; 28: 204-207.

12. Wye P, Bowman J, Wiggers J, Baker A, Knight J, Carr V, et al. Total smoking bans in psychiatric inpatient services : a survey of perceived benefits, barriers and support among staff. 2010; 10: 372.

13. Negative Effects of a Smoking Ban on an Inpatient Psychiatry Service Psychiatric Services.

14. Exposure to environmental tobacco smoke (ETS) and determinants of support for complete smoking bans in psychiatric settings. 2004; 13: 180-185.

15. Hall S. Treatment of Tobacco Use in an Inpatient Psychiatric Setting. 2015 55: $1265-1270$

16. Ansari W El, Stock C. Factors Associated With Smoking, Quit Attempts and Attitudes towards Total Smoking Bans at University: A Survey of Seven Universities in England, Wales and Northern Ireland. 2012; 13: 705-714.

17. Summary E, July I, Ban T. Indiana Smoking Ban in Effect. 1-3.

18. Ratschen E, Britton J, Mcneill A. Implementation of smoke-free policies in mental health in-patient settings in England. 2009; 547-551.

19. Cormac I, Mcnally L. How to implement a smoke-free policy †. 2008; 14 198-207.

20. Banham L, Gilbody S. Guest editorial. A smoking ban in psychiatric units threat or opportunity ? 2008; 129-134. 